\title{
A semi-automated system for quantifying the oxidative potential of ambient particles in aqueous extracts using the dithiothreitol (DTT) assay: results from the Southeastern Center for Air Pollution and Epidemiology (SCAPE)
}

\author{
T. Fang ${ }^{1}$, V. Verma ${ }^{1}$, H. Guo ${ }^{1}$, L. E. King ${ }^{1}$, E. S. Edgerton ${ }^{2}$, and R. J. Weber ${ }^{1}$ \\ ${ }^{1}$ School of Earth and Atmospheric Sciences, Georgia Institute of Technology, Atlanta, GA, USA \\ ${ }^{2}$ Atmospheric Research and Analysis, Inc., Cary, NC, USA
}

Correspondence to: R. J. Weber (rodney.weber@eas.gatech.edu)

Received: 17 May 2014 - Published in Atmos. Meas. Tech. Discuss.: 17 July 2014

Revised: 15 December 2014 - Accepted: 17 December 2014 - Published: 29 January 2015

\begin{abstract}
A variety of methods are used to measure the capability of particulate matter (PM) to catalytically generate reactive oxygen species (ROS) in vivo, also defined as the aerosol oxidative potential. A widely used measure of aerosol oxidative potential is the dithiothreitol (DTT) assay, which monitors the depletion of DTT (a surrogate for cellular antioxidants) as catalyzed by the redox-active species in PM. However, a major constraint in the routine use of the DTT assay for integrating it with large-scale health studies is its labor-intensive and time-consuming protocol. To specifically address this concern, we have developed a semi-automated system for quantifying the oxidative potential of aerosol liquid extracts using the DTT assay. The system, capable of unattended analysis at one sample per hour, has a high analytical precision (coefficient of variation of $15 \%$ for positive control, $4 \%$ for ambient samples) and reasonably low limit of detection $\left(0.31 \mathrm{nmol} \mathrm{min}{ }^{-1}\right)$. Comparison of the automated approach with the manual method conducted on ambient samples yielded good agreement (slope $=1.08 \pm 0.12$, $\left.r^{2}=0.92, N=9\right)$. The system was utilized for the Southeastern Center for Air Pollution \& Epidemiology (SCAPE) to generate an extensive data set on DTT activity of ambient particles collected from contrasting environments (urban, roadside, and rural) in the southeastern US. We find that water-soluble $\mathrm{PM}_{2.5}$ DTT activity on a per-air-volume basis was spatially uniform and often well correlated with $\mathrm{PM}_{2.5}$ mass ( $r=0.49$ to 0.88 ), suggesting regional sources contributing to the PM oxidative potential in the southeastern US. The correlation may also suggest a mechanistic ex-
\end{abstract}

planation (oxidative stress) for observed $\mathrm{PM}_{2.5}$ mass-health associations. The heterogeneity in the intrinsic DTT activity (per-PM-mass basis) across seasons indicates variability in the DTT activity associated with aerosols from sources that vary with season. Although developed for the DTT assay, the instrument can also be used to determine oxidative potential with other acellular assays.

\section{Introduction}

Epidemiological studies have associated increases in particulate matter (PM) levels with exacerbation of cardiovascular diseases (Zanobetti et al., 2014; Sun et al., 2010; Pope et al., 2004; Samet et al., 2000) and elevated incidence of respiratory disorders such as airway inflammation, bronchial muscle contraction, and asthma (Harkema et al., 2004; Schaumann et al., 2004; Aust et al., 2002; Norris et al., 1999). The mechanisms underlying these associations are not entirely clear, but reactive oxygen species (ROS) have been identified as a class of molecules that induce oxidative stress, causing cell damage (Nel, 2005; Gurgueira et al., 2002). ROS can either be adsorbed on inhaled particles or generated in vivo by target cells such as airway epithelial cells and macrophages (Li et al., 2003; Nel et al., 1998). These findings imply that aerosol oxidative potential, i.e., the ability of ambient particles to generate ROS, may be a more relevant measurement than PM mass concentration, or concentrations of specific aerosol 
chemical components, when attempting to link aerosols and health end points.

Both cellular (Xia et al., 2006; Kubátová et al., 2006; Bonvallot et al., 2001; Hiura et al., 1999; Antonini et al., 1998) and acellular methods (Zomer et al., 2011; Mudway et al., 2011; Jung et al., 2006; Cho et al., 2005; Venkatachari et al., 2005; Frampton et al., 1999) have been developed to measure the oxidative potential of PM. Both types of methods have advantages and disadvantages. Acellular methods require less-controlled environments and provide faster readouts of PM oxidative potential. One of the most widely used cell-free measures of particles oxidative potential is the DTT (dithiothreitol, $\left.\mathrm{HSCH}_{2}(\mathrm{CH}(\mathrm{OH}))_{2} \mathrm{CH}_{2} \mathrm{SH}\right)$ ) assay (Charrier and Anastasio, 2012; Verma et al., 2012, 2009a; Cho et al., 2005; Kumagai et al., 2002; Delfino et al., 2013). Typically, ROS in vivo are mainly produced in mitochondria and endoplasmic reticulum (ER) where molecular oxygen $\left(\mathrm{O}_{2}\right)$ are reduced to superoxide anion $\left(\mathrm{O}_{2}^{-}\right)$by accepting electrons from cellular reductants, such as NADPH, or during the ER protein folding process (Alfadda and Sallam, 2012). The DTT assay simulates this electron-transfer mechanism based on the catalytic ability of redox-active species to transfer electrons from DTT to oxygen, and thus can be considered a surrogate measure of the in vivo capacity of PM to induce ROS. The rate of the reaction, commonly called DTT activity, is determined by measuring the consumption of DTT over time, which is proportional to the concentration of redox-active species in PM extracts. Researchers have identified various chemical components that may participate in the reaction, including polycyclic aromatic hydrocarbons (PAHs) (Li et al., 2003), quinones (Chung et al., 2006; Kumagai et al., 2002), transition metals (Charrier and Anastasio, 2012), water-soluble organic carbon (WSOC) (Verma et al., 2009a; Cho et al., 2005), and HUmic-LIke Substances (HULIS) (Verma et al., 2012; Lin and Yu, 2011). However, a consensus on the relative contributions of these species in the overall DTT activity of ambient particles is currently lacking.

Studies have also reported the association between DTT activity of ambient particles and cellular biomarkers such as fractional exhaled nitric oxide $\left(\mathrm{FE}_{\mathrm{NO}}\right)-\mathrm{a}$ marker of airway inflammation (Delfino et al., 2013); heme oxygenase (HO-1) - an enzyme responsive to oxidative stress (Li et al., 2003); and 3-(4,5-dimethylthiazol-2-yl)2,5-diphenyltetrazolium bromide (MTT)-reduction activity (Steenhof et al., 2011). These studies suggest a plausible mechanistic link between DTT-assessed oxidative potential and adverse health effects of ambient particles. Routine DTT analysis as part of large-scale toxicological and health studies involving aerosol exposure is needed to further establish the health relevance of the DTT assay (and other chemical assays in general).

One major limitation in conducting the DTT assay on a large scale is its laborious and time-intensive analytical protocol, which requires precise handling practices at each step of the reaction for an accurate determination of the rate of
DTT oxidation. It generally takes at least $5 \mathrm{~h}$ for a two-person team to analyze five samples manually, limiting the total number of samples that can be analyzed daily. Researchers have also developed alternative approaches to the traditional DTT protocol (Cho et al., 2005) for new applications. For example, a paper-based analytical device ( $\mu$ PAD) DTT assay was developed for personal exposure studies (Sameenoi et al., 2012b), and a microfluidic electrochemical sensor coupled with a particle-into-liquid sampler (PILS) was developed for real-time measurement of aerosol DTT activity (Koehler et al., 2014; Sameenoi et al., 2012a). We have developed a semi-automated system using programmable syringe pumps with selector valves for conducting the DTT assay on various extracts. The system is based on a simplified protocol in which the aerosol extract oxidizes DTT in a single vial. A small aliquot is withdrawn at various time intervals to determine the remaining DTT concentration and calculate the rate of DTT consumption. Particulate samples are extracted to an aqueous state and analyzed in batches using an autosampler at roughly $1 \mathrm{~h}$ per sample. The semi-automated system can run for $24 \mathrm{~h}$ unattended and can also be monitored remotely. Extraction liquids can be either deionized water or organic solvents; in the latter case the solvent is evaporated and reconstituted in deionized water. The system was validated against the manual protocol (Cho et al., 2005) using both positive controls and ambient filter samples. Detailed comparisons of the semi-automated system and traditional manual method are shown in Table S1 in the Supplement. The DTT activity of more than 500 samples collected from the southeastern United States as part of the Southeastern Center for Air Pollution and Epidemiology (SCAPE) were analyzed for this study. Here we provide a detailed description and characterization of the automated system and an overview of aerosol oxidative potential in the southeastern United States. Sources and components of $\mathrm{PM}_{2.5}$ that produce the DTT activity are presented in Verma et al. (2014).

\section{Methods}

\subsection{Chemicals}

Dithiothreitol (DTT), Tris base, dimethyl sulfoxide (DMSO), 5,5'-dithiobis-(2-nitrobenzoic acid) (DTNB), and 9,10phenanthraquinone (PQN) were obtained from Sigma Aldrich (St. Louis, MO, USA). Trichloroacetic acid (TCA, $10 \% w / v$ ) was obtained from LabChem Inc. (Pittsburgh, PA, USA). Monopotassium phosphate $\left(\mathrm{KH}_{2} \mathrm{PO}_{4}\right)$, dipotassium phosphate $\left(\mathrm{K}_{2} \mathrm{HPO}_{4}\right)$, and ethylenediaminetetraacetate (EDTA) were obtained from VWR International LLC (West Chester, PA, USA). Details of the chemical preparation and storage are provided in the Supplement. 


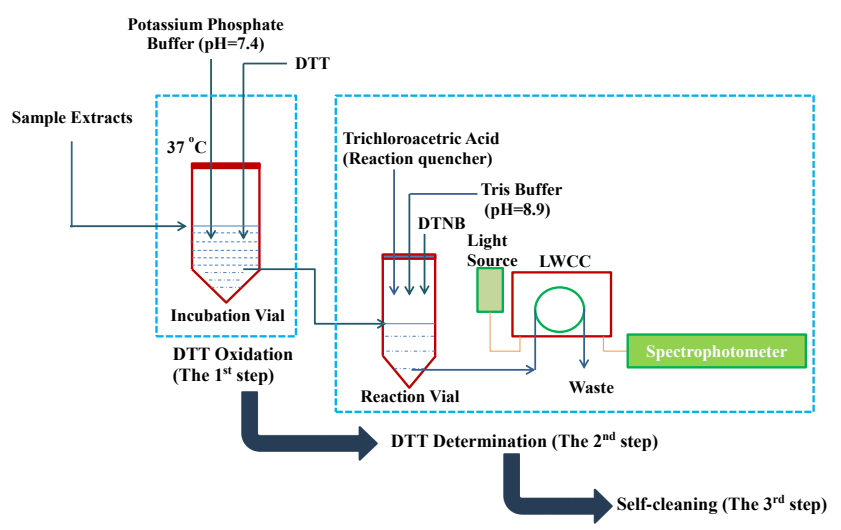

Figure 1. Automated system protocol.

\subsection{Automated DTT assay system development}

\subsubsection{Automated DTT assay system}

Illustrated protocol and setup schematic of the semiautomated DTT assay system are shown in Figs. 1 and 2, respectively. There are three steps in the automated DTT method. In the first step (DTT oxidation step), $3.5 \mathrm{~mL}$ of the aerosol extract sample and $1 \mathrm{~mL}$ potassium phosphate buffer $(0.5 \mathrm{mM})$ are loaded into an incubation vial (Figs. 1 and 2$)$ (sterile polypropylene centrifuge tube, VWR International LLC, Suwanee, GA, USA) using a programmable syringe pump (Fig. 2, pump A) (Kloehn, Inc., Las Vegas, NV, USA). Then $0.5 \mathrm{~mL}$ of DTT $(1 \mathrm{mM})$ is added to the incubation vial using another programmable syringe pump (Fig. 2, pump B). The DTT-buffer-sample mixture is incubated at $37^{\circ} \mathrm{C}$ and continuously shaken at a rotational frequency of $400 \mathrm{rpm}$ via a ThermoMixer (incubating accuracy: $\pm 0.5^{\circ} \mathrm{C}$; Eppendorf North America, Inc., Hauppauge, NY, USA). DTT in the mixture is consumed over time in the incubation vial due to catalytic reaction of DTT-active components of the aerosol extract.

In the second step (DTT determination step), at a specified time $(4 \mathrm{~min})$ following the completion of step one, a small aliquot $(100 \mu \mathrm{L})$ of the incubated mixture is withdrawn and transferred to another centrifuge tube (referred to as "reaction vial", wrapped in aluminum foil to prevent possible light interference), using pump B. This is mixed with $1 \mathrm{~mL}$ TCA ( $1 \% \mathrm{w} / v$; the quenching agent), which had previously been added to the reaction vial by pump A. The quenched mixture with residual DTT is further mixed with $2 \mathrm{~mL}$ of Tris buffer $(0.08 \mathrm{M}$ with $4 \mathrm{mM}$ EDTA $)$ and $0.5 \mathrm{~mL}$ DTNB $(0.2 \mathrm{mM})$ added by pump A. Reaction between the residual DTT and DTNB forms a light-absorbing product, 2-nitro-5-thiobenzoic acid (TNB), which has a high extinction coefficient of $14150 \mathrm{M}^{-1} \mathrm{~cm}^{-1}$ at $412 \mathrm{~nm}$ wavelength. Pump A then withdraws the final mixture from the reaction vial and pushes it through a Liquid Waveguide Capillary Cell (LWCC-M-100; World Precision Instruments, Inc., FL,

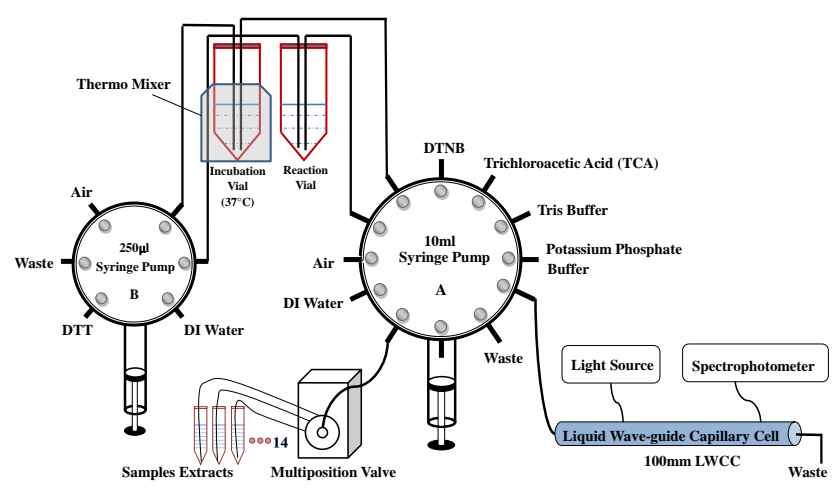

Figure 2. Automated system setup.

USA) with an optical path length of $100 \mathrm{~mm}$. The waveguide is coupled to an online spectrophotometer (Ocean Optics, Inc., Dunedin, FL, USA), which included a ultravioletvisible (UV-Vis) light source (Ocean Optics DT-Mini-2), and a multi-wavelength light detector (USB4000 Miniature Fiber Optic Spectrometer). Absorbance intensity at 412 and $700 \mathrm{~nm}$ (chosen as the baseline absorbance for TNB) are recorded every $2 \mathrm{~s}$ using data acquisition software (SpectraSuite). The system then performs a self-cleaning using deionized water (DI water, $>18 \mathrm{M} \Omega \mathrm{cm}^{-1}$ ) to eliminate any residual liquid in the reaction vial, tubing, syringes, and LWCC. This second step is repeated four more times, at specific time intervals $(13,23,30$ and $41 \mathrm{~min})$, generating a total of 5 data points of remaining DTT concentration with time. Following this, the automated system again performs a self-cleaning routine (third step) to ensure no carry-over in the incubation vial, tubing, syringes, and LWCC, before analyzing the next aerosol sample. A 14-port multiposition valve $\left(\mathrm{VICI}^{\circledR}{ }^{\circledR}\right.$ Valco Instrument Co. Inc., USA) is used to consecutively select individual aerosol samples for analysis. The Kloehn control program code written for conducting the DTT assay, including hardware and software details, is included in the Supplement. The automated system is cleaned periodically (about every 15 days) by flushing at least three times with methanol, followed by six times with DI water.

\subsubsection{DTT activity determination}

Figure 3 shows an example of the absorbance intensity plot measured over time for a filter blank (panel a) and sample (panel b), obtained from the data acquisition software. The time intervals $(4,13,23,30$, and $41 \mathrm{~min})$ represent the incubation duration of DTT and sample in potassium phosphate buffer for each measurement. Withdrawal of the mixture containing TNB and pushing it through the LWCC (DTT determination step) causes the corresponding jumps in light absorbance at $412 \mathrm{~nm}\left(\mathrm{~A}_{1}, \mathrm{~A}_{2}, \mathrm{~A}_{3}, \mathrm{~A}_{4}, \mathrm{~A}_{5}\right.$ in Fig. 3a). After the absorbance measurement, pump A pushes DI water through the LWCC, which returns the absorbance back to the baseline value (i.e., absorbance at $412 \mathrm{~nm}$ equals 

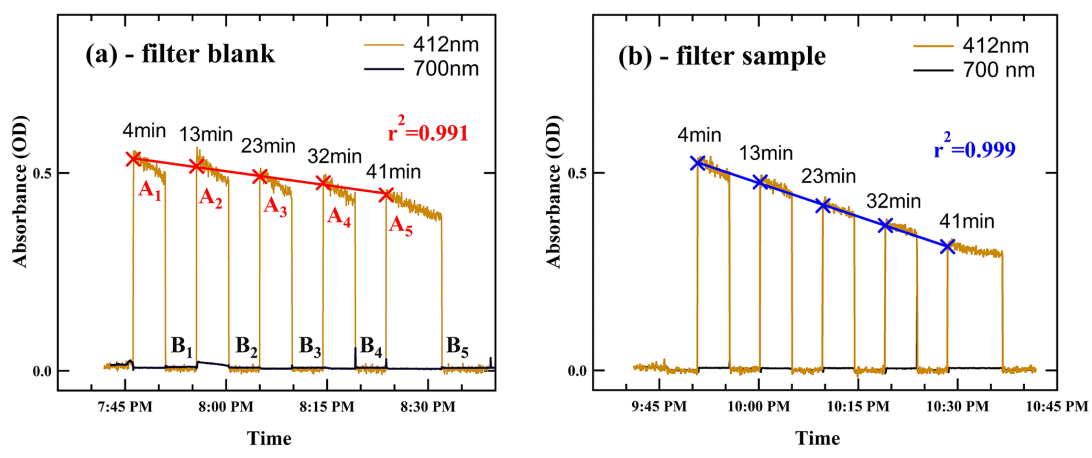

Figure 3. Example of an absorbance plot for a filter blank (a) and an ambient aerosol sample (b).

absorbance at $700 \mathrm{~nm} ; \mathrm{B}_{1}, \mathrm{~B}_{2}, \mathrm{~B}_{3}, \mathrm{~B}_{4}, \mathrm{~B}_{5}$ in Fig. 3a), thus generating a series of roughly square bars. A decreasing absorption intensity between successive measurements $\left(A_{1}>A_{2}>A_{3}>A_{4}>A_{5}\right)$ for a sample reflects the DTT oxidation over time. During each specified DTT measurement time interval, absorbance decreases (angled top of square wave in Fig. 3); the average of the initial five absorbance data is taken as characteristic absorption for each interval. The rate of DTT consumption ( $\sigma$ DTT, nmol min $^{-1}$ ) was determined from the slope and intercept of linear regression of measured absorbance versus time as follows:

$\sigma \mathrm{DTT}=-\sigma \mathrm{Abs} \cdot \frac{N_{0}}{\mathrm{Abs}_{0}}$,

where $\sigma \mathrm{Abs}$ is the slope of absorbance versus time; $\mathrm{Abs}_{0}$ is the initial absorbance calculated from the intercept of the linear regression of absorbance versus time; and $N_{0}$ is the initial moles of DTT added in the reaction vial. A steeper slope corresponds to a higher rate of DTT consumption. The final DTT activity for a sample was calculated by subtracting a blank value from the sample and normalized by sample air volume or particle overall mass (or mass of a specific component) represented by the sample in the incubation vial, expressed in the units of nmol $\mathrm{min}^{-1} \mathrm{~m}^{-3}$ for volumenormalized DTT activity (DTTv), or $\mathrm{nmol} \mathrm{min}^{-1} \mu \mathrm{g}^{-1}$ for mass-normalized DTT activity (DTTm). DTTm represents the intrinsic property of particles linked to sources, while DTTV accounts for the emission rate, dilution, etc., and characterizes exposure to the aerosol. Thus,

DTT activity $=\frac{\sigma \mathrm{DTT}_{\text {sample }}-\sigma \mathrm{DTT}_{\text {blank }}}{V_{\text {air }}\left(\text { or } M_{\text {particle }}\right)}$,

where $V_{\text {air }}$ and $M_{\text {particle }}$ are the ambient air volume $\left(\mathrm{m}^{3}\right)$ and total PM mass $(\mu \mathrm{g})$ represented by the sample in the incubation vial, respectively. For example, the total PM mass in the incubation vial for a sample with liquid concentration of $40 \mu \mathrm{g} \mathrm{mL}^{-1}$ would be $140 \mu \mathrm{g}\left(3.5 \mathrm{~mL} \times 40 \mu \mathrm{g} \mathrm{mL}^{-1}\right)$.

\subsection{Ambient sample collection and preparation}

\subsubsection{Sampling}

For this study, ambient $\mathrm{PM}_{2.5}$ samples were collected over $23 \mathrm{~h}$ on prebaked quartz filters (Pallflex ${ }^{\circledR}$ Tissuquartz $^{\mathrm{TM}}$, $8 \times 10$ inches) using high-volume (Hi-Vol) samplers (Thermo Anderson, flow rate normally $1.13 \mathrm{~m}^{3} \mathrm{~min}^{-1}$ ) as part of SCAPE. One sampler was fixed at a stationary site, Jefferson Street (JST), a central site representative of the Atlanta urban environment, while the other sampler (Trailer) was rotated among a roadside (RS), a near-road (Georgia Tech - GT), and a rural site (Yorkville - YRK), sampling in parallel with the fixed monitoring station (JST). Site characteristics are as follows:

1. RS site was situated within $5 \mathrm{~m}$ of the interstate highway (I75/85) in midtown Atlanta and was chosen for capturing immediate traffic emissions;

2. GT site was located on the rooftop of the Ford Environmental Science and Technology building at Georgia Tech, Atlanta, roughly $30 \mathrm{~m}$ above ground level, $840 \mathrm{~m}$ from the RS site, providing an intermediate location between RS and the central urban site (JST);

3. YRK site was situated in an agricultural region located approximately $70 \mathrm{~km}$ west of JST, representative of a rural environment.

Other sites also include an urban site in Birmingham (BHM), Alabama, and its paired rural site, Centerville (CTR), Alabama. JST, YRK, BHM, and CTR are all part of the Southeastern Aerosol Research and Characterization Study (SEARCH) network sites (Hansen et al., 2003). A map of sampling sites is provided in the Supplement. Ambient particles were collected from June 2012 to September 2013. For two periods (November 2012 and April 2013), the trailer was located at the stationary JST site for side-by-side comparisons. Table 1 provides the sampling schedule and number of filters collected at each site. In total, $503 \mathrm{Hi}$-Vol filters were collected over 15 months. Three seasons are defined based 
Table 1. Sampling schedule and number of Hi-Vol filters collected at each site from June 2012 to September 2013.

\begin{tabular}{lcccc}
\hline Year/month season & Stationary site & Sample size & Trailer site & Sample size \\
\hline 2012/Jun-Jul summer & JST & 31 & YRK & 33 \\
2012/Jul-Aug summer & JST & 37 & GT & 38 \\
2012/Sep-Oct fall & JST & 26 & RS & 29 \\
2012/Nov & JST & 13 & JST & 14 \\
2012/Dec winter & JST & 22 & YRK & 22 \\
2013/Jan-Feb winter & JST & 30 & RS & 31 \\
2013/Mar winter & JST & 23 & GT & 22 \\
2013/Apr & JST & 14 & JST & 14 \\
2013/Jun-Jul summer & CTR & 30 & BHM & 31 \\
2013/Sep-Oct fall & GT & 23 & RS & 20 \\
\hline Total number of filters & & & & 503 \\
\hline
\end{tabular}

on the temperature profile throughout sampling period (see Supplement, Fig. S2) - summer, fall, and winter, as shown in Table 1. Collected samples were immediately wrapped in prebaked aluminum foil and stored at $-18^{\circ} \mathrm{C}$ until analyzed.

\subsubsection{Extraction of PM filters}

All filters extracted in DI water via the following method were also extracted in methanol, dried, and reconstituted in DI water for DTT analysis. Both water and methanol extracts were separated by solid-phase extraction (C-18 resin). DTT activity was analyzed with this system in order to explore the oxidative potential of chemical sub-fractions of the ambient aerosol. Thus, not including blanks and positive controls, for this project on the order of 2000 extracts were analyzed for DTT activity. For the water extraction procedure, portions of the filters (about $1 / 28$ of the area of the $\mathrm{Hi}$-Vol filter, three 1-inch-diameter punches) were punched and extracted in $15 \mathrm{~mL}$ of DI water in sterile polypropylene centrifuge tubes (VWR International LLC, Suwanee, GA, USA) by sonication using an Ultrasonic Cleanser (VWR International LLC, West Chester, PA, USA) for $30 \mathrm{~min}$. Extracts were then filtered using PTFE $0.45 \mu \mathrm{m}$ syringe filters (Fisherbrand $^{\mathrm{TM}}$, Fisher Scientific, PA, USA) to remove insoluble components, and divided into two fractions. One fraction of at least $5 \mathrm{~mL}$ was analyzed for DTT activity using the automated system. The other fraction was reserved for other chemical analysis including water-soluble organic carbon and brown carbon. Here we focus only on the discussion of DTT analysis of the direct water extracts.

\section{$2.4 \quad \mathbf{P M}_{2.5}$ mass concentration}

$\mathrm{PM}_{2.5}$ mass concentration were measured by a Tapered Element Oscillating Microbalance (TEOM) at the SEARCH sites - i.e., JST, YRK, BHM, and CTR by Atmospheric Research Analysis (ARA, Inc.) - and retrieved from the data archive. For the RS and GT sites, the PM mass concentrations were estimated from the sum of chemical com- ponents analyzed on the Hi-Vol filters and the summation method was calibrated with ARA TEOM at the JST site (slope $=1.34 \pm 0.06$, intercept $=-2.46 \pm 0.59 \mu \mathrm{g} \mathrm{m}^{-3}$, $r^{2}=0.72, N=162$; see Supplement, Fig. S3). The measured components include elemental carbon (EC; Sunset Laboratory OCEC analyzer), organic mass (OC $\cdot 1.6$; Turpin and Lim, 2001), water-soluble metals (measured by X-ray fluorescence method, XRF), and ammonium sulfate (assuming sulfate and ammonium are all $\left(\mathrm{NH}_{4}\right)_{2} \mathrm{SO}_{4}$ (Zhang et al., 2010), where sulfate was calculated from sulfur measured by XRF). Further description of the analytical procedure for each method will be reported in subsequent publications on the chemical data (Verma et al., 2014; Fang et al., 2015).

\section{Results and discussion}

\subsection{Automated system performance}

Performance of the automated system was assessed by conducting tests to determine instrument response, limit of detection (LOD), precision, and accuracy using both positive controls and ambient particles.

\subsubsection{Automated system response to individual compound}

PQN has been shown to be capable of catalyzing the oxidation of DTT (Li et al., 2009; Kumagai et al., 2002), although it is not highly water-soluble. The automated system was assessed for linearity with PQN (Fig. 4). The $x$ axis intercept $\left(0.11 \mathrm{nmol} \mathrm{mL}^{-1}\right.$ in the incubation vial) represents the minimum concentration of $\mathrm{PQN}$ required to produce a measurable signal on the system with a pure water blank subtracted. As shown, the response of the system to PQN is highly linear with a correlation coefficient $\left(r^{2}\right)$ of 0.95 . At least one PQN per ambient sample batch (typically 12 samples) is used as a positive control to ensure the consistency of the system. 


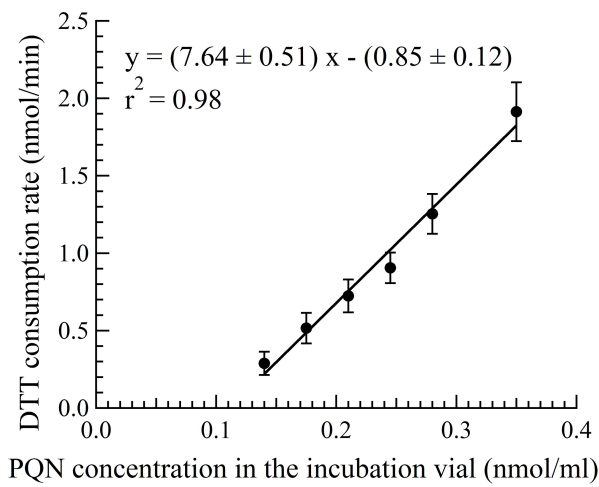

Figure 4. Blank corrected DTT consumption rate as a function of PQN (9,10-phenanthraquinone) used as a positive control. Each error bar represents the standard deviation of three independent DTT measurements on each concentration.

\subsubsection{Limit of detection}

The limit of detection (LOD) of the system, defined as 3 times the standard deviation of DI water blanks $(N=37)$,

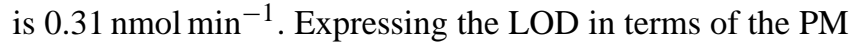
concentration of the sample extract $\left(\mu \mathrm{g} \mathrm{mL} L^{-1}\right)$ is not straightforward as it depends on many factors, including extraction efficiency and the relative fractions of DTT-active components in ambient particles. Based on the analysis of $503 \mathrm{Hi}-$ Vol samples in the present study from different sites (urban, rural, and roadside), approximately $100 \mu \mathrm{g}$ of $\mathrm{PM}_{2.5}$ mass loading on the filter, extracted in $5 \mathrm{~mL}$ of DI water, was sufficient to have reliable results above LOD. The upper limit of the PM concentration is also constrained such that the DTT consumption remains less than $50 \%$ of the initial concentration to ensure the pseudo-1st-order reaction of the DTT oxidation. For most of the PM samples collected, roughly $1 / 28$ of the area (typical $\mathrm{PM}_{2.5}$ mass loading $=0.2-1 \mathrm{mg}$ ) of a HiVol filter, extracted in $15 \mathrm{~mL}$ of DI water, yielded a DTT consumption rate within these limits $\left(0.8-2.6 \mathrm{nmol} \mathrm{min}^{-1}\right)$. In rare cases $(<10 \%)$ of the DTT consumption exceeding $50 \%$, only the initial consumption data points (at least 3 ) were used for the rate calculation.

\subsubsection{Precision}

The precision of the automated system for ambient samples was assessed by separately extracting seven different equal sections $(1 / 28$ each) of the same Hi-Vol filter in $15 \mathrm{~mL}$ of DI water and analyzed for DTT activity. A low standard deviation of $0.081 \mathrm{nmol} \mathrm{min}^{-1}$ (coefficient of variation, $\mathrm{CV}=4 \%$ ) indicates sufficiently high precision of the system for ambient samples.

\subsubsection{Accuracy}

The system was validated for accuracy by comparing the DTT activity of both positive controls and ambient PM sam-

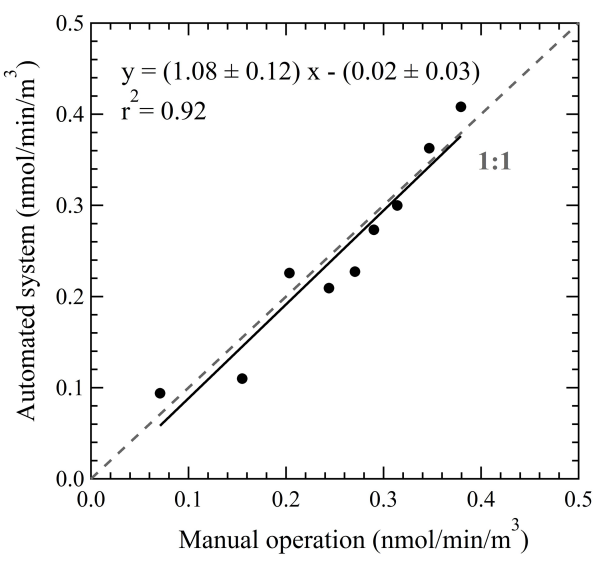

Figure 5. Comparison of the automated system with manual operation using ambient aerosol extracts $\left(\mathrm{PM}_{2.5}\right.$ samples collected from JST site, Atlanta, in December 2012) (regression analysis was done by orthogonal regression; the dotted line is $1: 1$ ).

ples obtained from the automated approach with that from the same experimental protocol performed manually (Cho et al., 2005).

Five replicates of the PQN solutions $\left(0.21 \mathrm{nmol} \mathrm{mL}^{-1}\right.$ in the incubation vial) were run both on the automated system and manually. The DTT consumption rate obtained from the automated system (mean $\pm \mathrm{SD}$ of $0.77 \pm 0.03 \mathrm{nmol} \mathrm{min}{ }^{-1}$, $\mathrm{CV}=4.24 \%)$ was very close to that from the manual operation $\left(0.74 \pm 0.03 \mathrm{nmol} \mathrm{min}^{-1}, \mathrm{CV}=3.97 \%\right)$. As further validation, nine ambient PM samples were analyzed for DTT activity by both manual and automated approach. As shown in Fig. 5, an orthogonal fit yielded a slope (automated/manual) of $1.08 \pm 0.12$, intercept close to 0 $\left(-0.02 \pm 0.03 \mathrm{nmol} \mathrm{min} \mathrm{m}^{-1} \mathrm{~m}^{-3}\right)$, and correlation coefficient $\left(r^{2}\right)$ of 0.92 . Further, a paired $t$ test shows no significant difference between the results obtained by the two methods $\left(t(8)<t_{\text {critical }}, p=0.05\right)$.

These tests demonstrate the robustness of the instrument as a viable alternative to the manual DTT assay, making it useful for rapid and high-throughput sample analysis for largescale studies.

\subsection{Field evaluation of the automated system}

SCAPE was a coordinated multi-investigator effort to characterize ambient gas-particle mixtures in the southeastern US, to elucidate their sources, and to assess their impacts on human health. The automated system was used to measure DTT activity on the set of samples $(N=503)$ collected from multiple sites during SCAPE. Multiple DI water blanks $(N=37)$ and PQN solutions $(N=55)$, in addition to field blanks $(N=63)$ collected during the sampling, were analyzed along with the PM samples. This project provided an ideal opportunity for the field evaluation of the semi-automated DTT assay instrument. Table 2 summarizes the performance of the sys- 
Table 2. Performance of the automated system as assessed by consistency of DTT consumption rate for blanks and positive controls.

\begin{tabular}{lccc}
\hline Sample & Sample size & Standard deviation, nmol/min & $\% \mathrm{CV}$ \\
\hline DI blank & 37 & 0.10 & 28.1 \\
Field blank extracted in DI water & 63 & 0.11 & 31.1 \\
Positive controls (9,10-phenanthrenequinone) & 55 & 0.19 & 15.0 \\
\hline
\end{tabular}

Coefficient of variation $(\% \mathrm{CV})=\mathrm{SD} / \mathrm{Avg} \cdot 100$

tem as evaluated by stability of the results from both positive controls and blanks. The system remained fairly consistent throughout the analysis with reasonably small variability for both positive controls $(\mathrm{CV}=15 \%)$ and blanks (DI blanks: $\mathrm{CV}=28.1 \%$; field blanks: $\mathrm{CV}=26.7 \%$ ).

\subsubsection{Overall method precision}

The overall precision of the paired ambient sampling method utilized in this study was further assessed by comparing the DTT activity of $\mathrm{PM}_{2.5}$ samples collected simultaneously at JST using two Hi-Vol samplers in November 2012 and April 2013 (shown in Fig. 6). The orthogonal regression yields a slope of $1.03 \pm 0.05$, with an intercept of $0.02 \pm 0.01 \mathrm{nmol} \mathrm{min}^{-1} \mathrm{~m}^{-3}$, and $r^{2}=0.96$. Considering the combined uncertainties from sample collection, pretreatment, and extraction, the good agreement between the two sampling systems demonstrates a high overall precision of DTT measurement for ambient samples.

\subsubsection{DTT activity of ambient samples}

The time series of both water-soluble, volume-normalized (DTTv) and water-soluble, mass-normalized (DTTm) DTT activity are shown in Fig. 7a and b, respectively. Comparing the time series of DTTv (Fig. 7a) between paired sites shows that there is generally little divergence between sites, with the exception of the JST-YRK (urban-rural) pair in winter. Some of this uniformity is due to the $23 \mathrm{~h}$ integrated sampling time of the Hi-Vol filters, which dampens any diurnal variability in the emission sources contributing to PM oxidative potential. Figure 8 shows the distribution of watersoluble DTTv and DTTm data of ambient $\mathrm{PM}_{2.5}$ obtained in our study in comparison with those from other studies (Charrier and Anastasio, 2012; Verma et al., 2009b; Hu et al., 2008; Ntziachristos et al., 2007; Cho et al., 2005). Our DTTv data (Fig. 8a) are in the range reported in other studies, with the exception of RS (measurements made adjacent to a high-traffic highway), which is lower in the present study. However, comparing the DTTm levels, RS DTTm are well within the typical range of other studies, and thus our lower DTTv levels are most probably attributed to higher $\mathrm{PM}_{2.5}$ concentrations reported in other studies sampling by roadways. Our study generally shows a broader span for urban and rural sites than reported previously, which is likely due to the much larger data set collected over a yearlong period

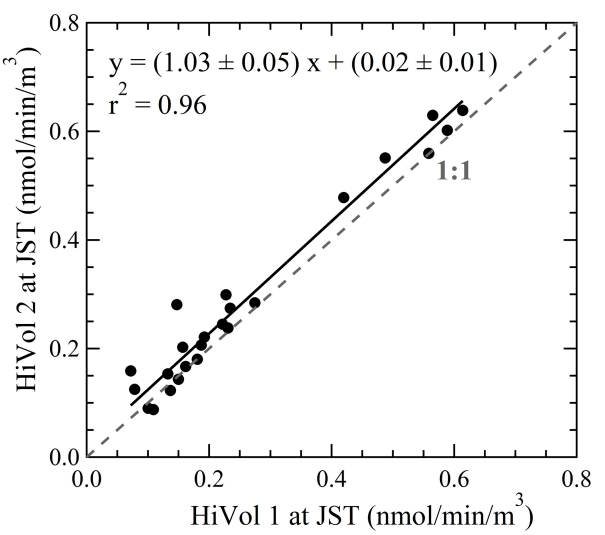

Figure 6. Overall method precision of the automated system assessed by $\mathrm{PM}_{2.5}$ filter samples $(N=24)$ collected simultaneously using two Hi-Vol samplers deployed side-by-side at JST during November 2012 and April 2013 (regression analysis was done by orthogonal regression; the dotted line is $1: 1$ ).

that captured a wider range of sources and ambient conditions.

\subsubsection{Seasonal and spatial variability}

The spatiotemporal variability of DTTv can be used to assess the exposure to total water-soluble DTT-active species in different seasons and sites. Since DTTm is represented by DTTv divided by $\mathrm{PM}_{2.5}$ mass concentration, which is the intrinsic property of aerosol independent of exposure levels, the spatiotemporal variability of DTTm provides information on the variation of DTT-active chemical components. Seasonal and spatial variability of aerosol oxidative potential (both DTTv and DTTm) in the southeastern US was assessed by analysis of variance (ANOVA) tests and coefficient of divergence (COD), respectively. The ANOVA tests were used to assess differences between seasons at a given site (Supplement, Tables S2 and S3), and the COD (Wilson et al., 2005) was calculated to assess spatial variability (see Table 3 ). The COD is calculated as follows:

$\mathrm{COD}=\sqrt{\frac{1}{N} \sum_{i=1}^{N}\left[\frac{c_{i j}-c_{i k}}{c_{i j}+c_{i k}}\right]^{2}}$,

where $c_{i j}$ and $c_{i k}$ are the $23 \mathrm{~h}$ averaged DTT activity $\left(\mathrm{nmol} \min ^{-1} \mathrm{~m}^{-3}\right.$ ) measured at site $j$ and $k$, respectively, and 

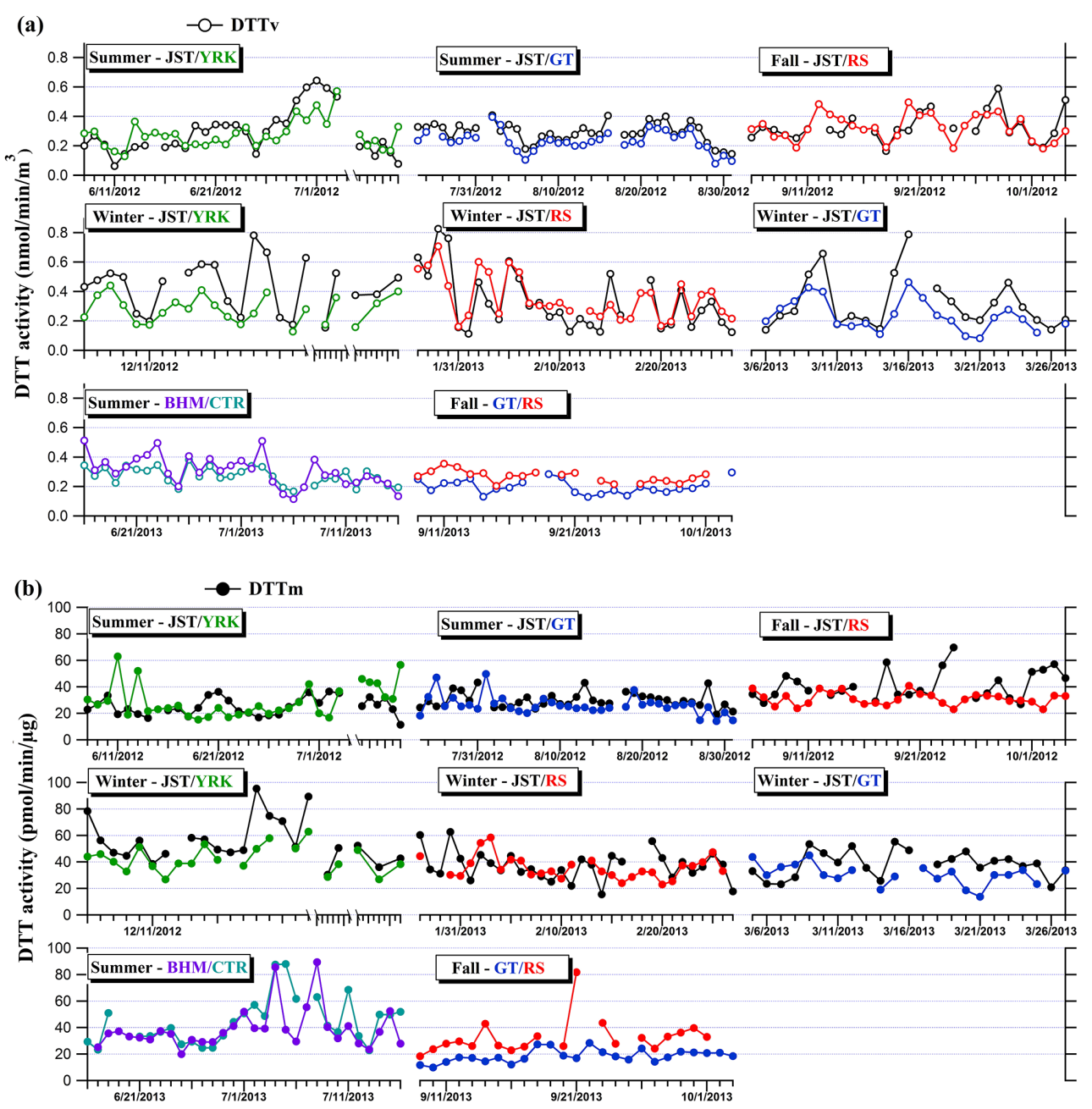

Figure 7. Water-soluble DTT activity of the ambient particles collected at paired sampling sites. The plots show (a) volume-normalized (DTTv) and (b) mass-normalized (DTTm) DTT activity at JST (urban, black) paired with YRK (rural, green), RS (roadside, red), and GT (near road, blue). Also included is the CTR (rural, cyan)-BHM (urban, purple) pair, and the GT-RS pair.

Table 3. Spatial heterogeneity of DTTv and DTTm assessed by coefficient of divergence (COD).

\begin{tabular}{llcc}
\hline Season & Paired sites & CODv (DTTv) & CODm (DTTm) \\
\hline Summer & JST-YRK & 0.21 & 0.23 \\
Summer & JST-GT & 0.15 & 0.15 \\
Fall & JST-RS & 0.13 & 0.23 \\
Winter & JST-YRK & 0.25 & 0.15 \\
Winter & JST-RS & 0.17 & 0.18 \\
Winter & JST-GT & 0.23 & 0.22 \\
Summer 2013 & BHM-CTR & 0.12 & 0.15 \\
Fall 2013 & GT-RS & 0.18 & 0.31 \\
\hline
\end{tabular}

$N$ is the sample size. COD ranges from 0 to 1 , with values close to 0 representing a homogenous distribution and those near 1 indicating heterogeneity.
Based on ANOVA tests, there was high heterogeneity across seasons for DTTv at JST ( $p=0.01$; see Supplement, Table S2), with the highest DTTv in winter (December (winter $) /$ June $($ summer $)=1.51)$, while there was no significant seasonal variation observed at YRK, GT, or the RS site $(p>0.01)$. In comparison to DTTv, there are greater seasonal variations in DTTm; for example, average DTTm at most sites showed much higher levels in winter than summer and fall (winter/summer $=1.4,1.2$, and 2.2 for YRK, GT, and JST, respectively). ANOVA tests also validated the pronounced seasonal differences for DTTv and DTTm at JST and DTTm at other sites ( $p<0.01$; see Supplement, Tables S2 and S3). The higher seasonal differences in DTTm may suggest that the specific chemical components that contribute to the oxidative potential of particles vary between seasons and originate from different sources.

Relatively low levels of the CODs $(<0.25)$ (Table 3$)$ found for both DTTv and DTTm at paired sites indicate spatial 
(a) - DTTV

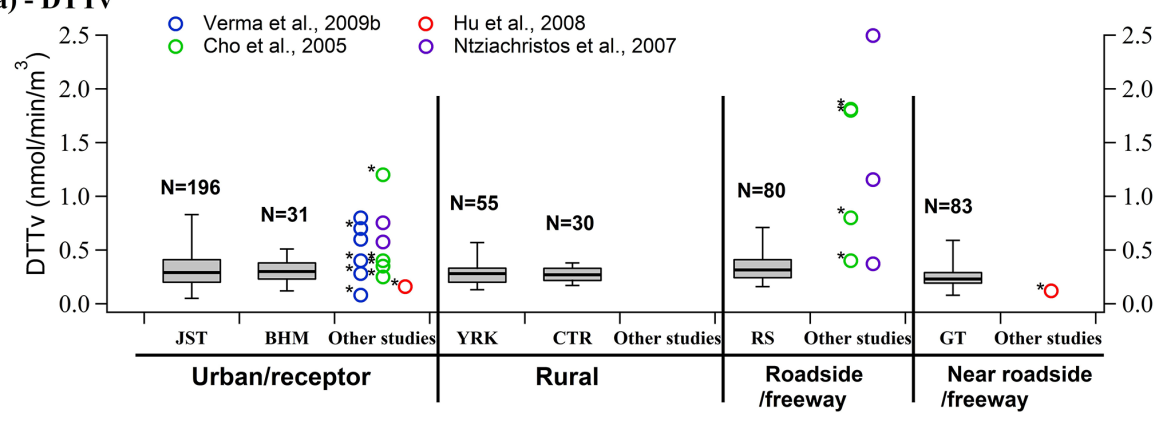

(b)

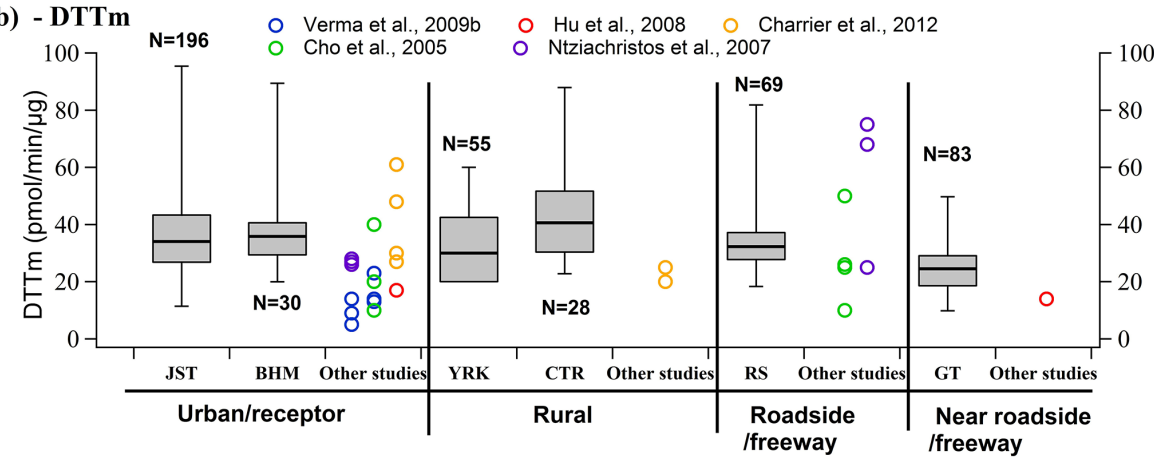

Figure 8. Distribution of water-soluble volume-normalized (a) and mass-normalized (b) DTT activity (oxidative potential) of ambient PM 2.5 in our study compared with other studies. Data from this study are expressed as median, maximal, minimal, and percentile 25 and 75. Roadside/freeway - sampling sites are located adjacent to road/highway; near roadside/freeway - sampling sites are further away but less than $1 \mathrm{~km}$ from road/freeway. ${ }^{*}$ - numbers not presented in the paper, thus estimated from graphs.

homogeneity of water-soluble aerosol oxidative potential in the region, suggesting dominant DTT activity sources are regional in nature, such as SOA (secondary organic aerosol) or a well-mixed atmosphere of emissions from distant sources (e.g., long-range transport). Note that JST-RS and GT-RS pairs, in both fall seasons, show slightly lower CODs (more uniformity) in DTTv than in DTTm (COD for DTTv $=0.13$ and 0.18 compared to COD for DTTm $=0.23$ and 0.31 for JST-RS and GT-RS, respectively). This indicates that, although there may be unique local sources at the RS site, for example primary vehicle emissions and re-suspended dust, their overall contribution was not substantial compared to the regional DTT signal and so was not clearly resolved.

\subsubsection{Oxidative potential and $\mathbf{P M}_{2.5}$ mass}

Figure 9 shows the correlation between DTT activity (DTTv) and $\mathrm{PM}_{2.5}$ mass concentrations for all the sites and seasons. DTTv correlates with PM concentration in various degrees (Pearson's $r=0.49-0.88$ ), but generally the correlations are high for most sites. For JST, the representative urban site, Pearson's $r$ is particularly high $(r=0.76-0.82)$ in all seasons. Lowest DTTv- $\mathrm{PM}_{2.5}$ mass correlations were at the roadside site, probably due to the influence of unique RS sources that contributed to mass but not significantly to DTT activity (average $\mathrm{PM}_{2.5}$ concentrations are 8.7 and
$8.2 \mu \mathrm{g} \mathrm{m}^{-3}$ at JST compared to 10.4 and $9.5 \mu \mathrm{g} \mathrm{m}^{-3}$ at RS in fall and winter, respectively). It is important to note that, despite the significant correlation, the slope for DTT activity versus $\mathrm{PM}_{2.5}$ mass varies among different sites and seasons. Overall, we attribute the variation in degree of association between DTT activity and $\mathrm{PM}_{2.5}$ mass to the varying PM chemical composition.

For example, DTT activity correlates with WSOC in summer $(R>0.67)$ but correlates better with brown carbon in winter $(R>0.78)$. Further investigation on identifying the specific sources and aerosol chemical components linked to PM oxidative potential was discussed in Verma et al. (2014).

Studies have shown a correlation between $\mathrm{PM}_{2.5}$ mass and health end points - such as heart rate variability (HRV), blood neutrophils, and lipid changes (Tong et al., 2012) - increased oxidative DNA damage and nucleobases (Peter and Steffen, 2010), and cardiovascular mortality (Gholampour et al., 2014). The overall correlation between water-soluble DTT activity and $\mathrm{PM}_{2.5}$ mass concentration observed in this study may help explain, at least in part, some of these associations. However the varying degree of correlation between DTT activity and $\mathrm{PM}_{2.5}$ mass at different sites and seasons suggests that there are additional advantages of including PM oxidative potential in health studies, rather than relying on PM mass alone. 


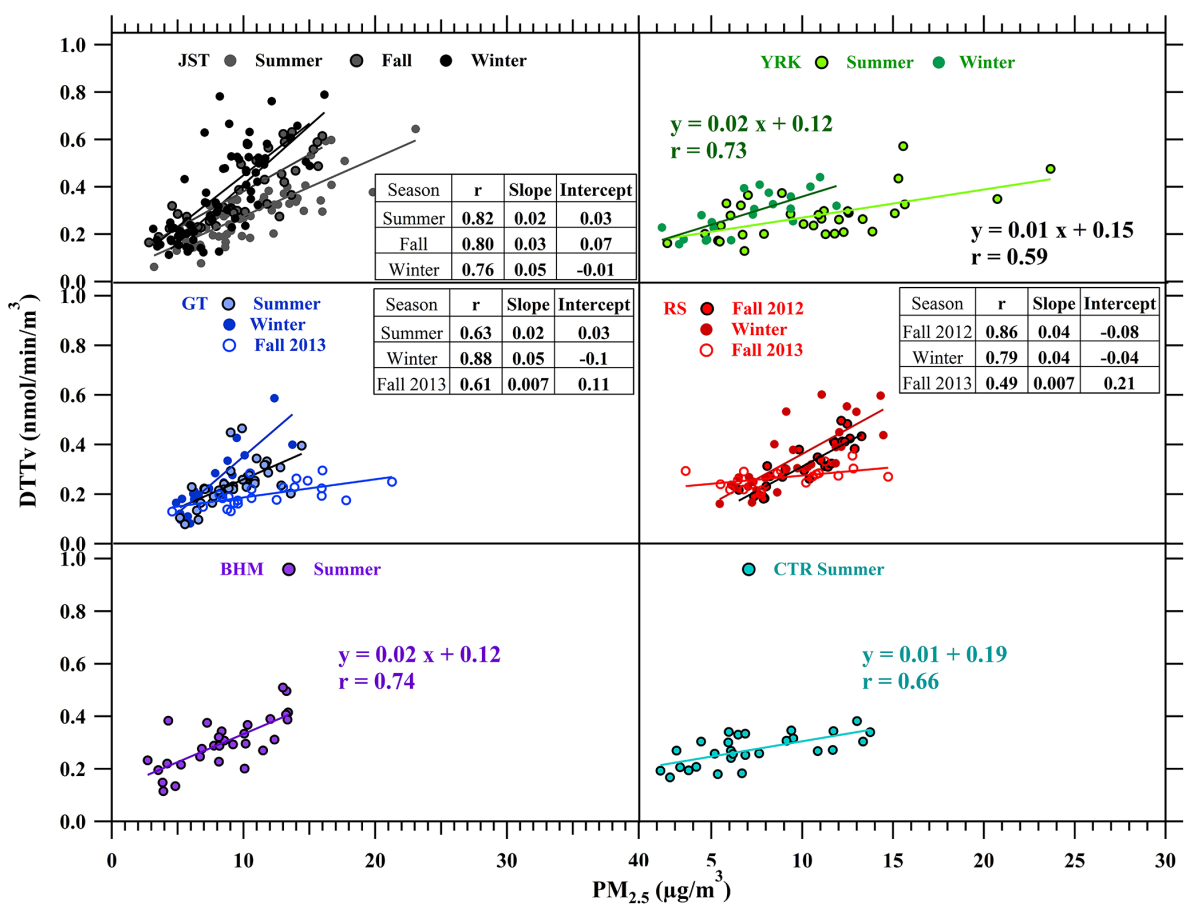

Figure 9. Correlation (Pearson's $r$ ) between water-soluble DTT activity (DTTv) and $\mathrm{PM}_{2.5}$ mass concentration at JST (urban), YRK (rural), RS (roadside), GT (near-road), CTR (rural), and BHM (urban) sites.

\section{Conclusions}

An automated analytical system for quantifying the oxidative potential of aerosol liquid extracts using the DTT assay was developed. The system follows the analytical approach developed by Cho et al. (2005) and is capable of one DTT activity measurement per hour. The system response was assessed by PQN, which was used as a positive control when running a series of ambient samples. The method LOD was $0.31 \mathrm{nmol} \mathrm{min}{ }^{-1}$. Analytical precision based on both PQN $(\mathrm{CV}=15 \%)$ and ambient samples $(\mathrm{CV}=4 \%)$ was high. The instrument was further validated for accuracy by comparing with the manual procedure using ambient PM samples $\left(r^{2}=0.92\right.$, slope $\left.=1.08 \pm 0.12\right)$. The suitability of the system for large-scale application was assessed by analyzing more than 500 filters collected in the southeastern US as part of SCAPE, the joint Emory-Georgia Tech Clean Air Research Center. The data show that water-soluble $\mathrm{PM}_{2.5}$ DTT activity on a per-volume-of-air basis was spatially uniform and generally correlated with $\mathrm{PM}_{2.5}$ mass $(r=0.49$ to 0.88), indicating DTT activity in the southeast is likely, to a significant extent, related to regional sources and not dominated by a single source or a limited number of species. However, the higher seasonal heterogeneity in the intrinsic water-soluble DTT activity (per-PM-mass basis) may indicate that the dominant regional sources change with season. More in-depth analysis of the extensive data set generated with the instrument will be forthcoming. It is noted that the method can be altered to run smaller sample volumes, for situations involving samples of lower mass loadings. It has also been modified for ROS analysis using other assays (e.g., ascorbate depletion assay). The automated system presents a useful new tool for rapid and high-throughput measurement of the DTT activity of ambient particle extracts. Its application can facilitate routine use of PM oxidative potential in toxicological, panel exposures, and epidemiological studies. More work is required to identify specific compounds that are most sensitive in the DTT probe and test how they may contribute to the observed ambient DTT activity. Application of the DTT probe and other acellular and cellular assays in different regions that have a different mix of emission sources is also needed to better understand the possible links between aerosol oxidative potential and health end points. The automated DTT assay analytical instrument described here would facilitate these types of future studies. Finally, although the analytical system can provide new insight on aerosol oxidative properties from large data sets, it does not solve limitations associated with filterbased particle collection approaches, which entail artifacts due to losses of semivolatile DTT-active species during sampling and handling procedures. Adapting the DTT assay to an online system could limit sampling artifacts and provide new insights into DTT sources and atmospheric processing that more highly time-resolved data can provide. 


\section{The Supplement related to this article is available online at doi:10.5194/amt-8-471-2015-supplement.}

Acknowledgements. We wish to thank the Southeastern Aerosol Research and Characterization (SEARCH) personnel for their many contributions supporting the field deployments. We also thank R. Erik Weber, Janessa Riana Rowland, and Madhusudan Kamat for assistance in lab work. This research was made possible by US EPA grant R834799. T. Fang acknowledges the support from the Oversea Study Program of Guangzhou Elite Project.

Disclaimer. The contents are solely the responsibility of the grantee and do not necessarily represent the official views of the US EPA. Further, US EPA does not endorse the purchase of any commercial products or services mentioned in the work.

Edited by: P. Herckes

\section{References}

Alfadda, A. A. and Sallam, R. M.: Reactive oxygen species in health and disease, J. Biomed. Biotechnol., 2012, 936486, doi:10.1155/2012/936486, 2012.

Antonini, J. M., Clarke, R. W., Krishna Murthy, G. G., Sreekanthan, P., Jenkins, N., Eagar, T. W., and Brain, J. D.: Freshly generated stainless steel welding fume induces greater lung inflammation in rats as compared to aged fume, Toxicol. Lett., 98, 77-86, 1998.

Aust, A. E., Ball, J. C., Hu, A. A., Lighty, J. S., Smith, K. R., Straccia, A. M., Veranth, J. M., and Young, W. C.: Particle characteristics responsible for effects on human lung epithelial cells, Res. Rep. Health Eff. Inst., 110, 1-65, 2002.

Bonvallot, V., Baeza-Squiban, A., Baulig, A., Brulant, S., Boland, S., Muzeau, F., Barouki, R., and Marano, F.: Organic compounds from diesel exhaust particles elicit a proinflammatory response in human airway epithelial cells and induce cytochrome p450 1A1 expression, Am. J. Resp. Cell Mol., 25, 515-521, 2001.

Charrier, J. G. and Anastasio, C.: On dithiothreitol (DTT) as a measure of oxidative potential for ambient particles: evidence for the importance of soluble transition metals, Atmos. Chem. Phys., 12, 9321-9333, doi:10.5194/acp-12-9321-2012, 2012.

Cho, A. K., Sioutas, C., Miguel, A. H., Kumagai, Y., Schmitz, D. A., Singh, M., Eiguren-Fernandez, A., and Froines, J. R.: Redox activity of airborne particulate matter at different sites in the Los Angeles Basin, Environ. Res., 99, 40-47, 2005.

Chung, M. Y., Lazaro, R. A., Lim, D., Jackson, J., Lyon, J., Rendulic, D., and Hasson, A. S.: Aerosol-borne quinones and reactive oxygen species generation by particulate matter extracts, Environ. Sci. Technol., 40, 4880-4886, 2006.

Delfino, R. J., Staimer, N., Tjoa, T., Gillen, D. L., Schauer, J. J., and Shafer, M. M.: Airway inflammation and oxidative potential of air pollutant particles in a pediatric asthma panel, J. Expos. Sci. Environ. Epidemiol., 23, 466-473, 2013.

Fang, T., Verma, V., Peltier, R. E., Guo, H. Y., King, L. E., Edgerton, E. S., and Weber, R. J.: Distribution of ambient atmospheric water-soluble metals in the southeastern United States and insights into their complexation states, in preparation, 2015.
Frampton, M. W., Ghio, A. J., Samet, J. M., Carson, J. L., Carter, J. D., and Devlin, R. B.: Effects of aqueous extracts of $\mathrm{PM}_{10}$ filters from the Utah Valley on human airway epithelial cells, Am. J. Phys.-Lung C., 277, L960-L967, 1999.

Gholampour, A., Nabizadeh, R., Naseri, S., Yunesian, M., Taghipour, H., Rastkari, N., Nazmara, S., Faridi, S., and Mahvi, A. H.: Exposure and health impacts of outdoor particulate matter in two urban and industrialized area of Tabriz, Iran, Journal of Environmental Health Science and Engineering, 12, 27, doi:10.1186/2052-336X-12-27, 2014.

Gurgueira, S. A., Lawrence, J., Coull, B., Murthy, G. G., and Gonzalez-Flecha, B.: Rapid increases in the steady-state concentration of reactive oxygen species in the lungs and heart after particulate air pollution inhalation, Environ. Health Persp., 110, 749-755, 2002.

Hansen, D. A., Edgerton, E. S., Hartsell, B. E., Jansen, J. J., Kandasamy, N., Hidy, G. M., and Blanchard, C. L.: The Southeastern Aerosol Research and Characterization Study: Part 1-Overview, J. Air Waste Ma., 53, 1460-1471, 2003.

Harkema, J. R., Keeler, G., Wagner, J., Morishita, M., Timm, E., Hotchkiss, J., Marsik, F., Dvonch, T., Kaminski, N., and Barr, E.: Effects of concentrated ambient particles on normal and hypersecretory airways in rats, Res. Rep. Health Eff. Inst., 120, 1-68, 2004.

Hiura, T. S., Kaszubowski, M. P., Li, N., and Nel, A. E.: Chemicals in diesel exhaust particles generate reactive oxygen radicals and induce apoptosis in macrophages, J. Immunol., 163, 5582-5591, 1999.

Hu, S., Polidori, A., Arhami, M., Shafer, M. M., Schauer, J. J., Cho, A., and Sioutas, C.: Redox activity and chemical speciation of size fractioned PM in the communities of the Los Angeles-Long Beach harbor, Atmos. Chem. Phys., 8, 64396451, doi:10.5194/acp-8-6439-2008, 2008.

Jung, H., Guo, B., Anastasio, C., and Kennedy, I. M.: Quantitative measurements of the generation of hydroxyl radicals by soot particles in a surrogate lung fluid, Atmos. Environ., 40, 1043-1052, 2006.

Koehler, K. A., Shapiro, J., Sameenoi, Y., Henry, C., and Volckens, J.: Laboratory evaluation of a microfluidic electrochemical sensor for aerosol oxidative load, Aerosol Sci. Technol., 48, 489497, 2014.

Kubátová, A., Dronen, L. C., Picklo, M. J., and Hawthorne, S. B.: Midpolarity and nonpolar wood smoke particulate matter fractions deplete glutathione in RAW 264.7 macrophages, Chem. Res. Toxicol., 19, 255-261, 2006.

Kumagai, Y., Koide, S., Taguchi, K., Endo, A., Nakai, Y., Yoshikawa, T., and Shimojo, N.: Oxidation of proximal protein sulfhydryls by phenanthraquinone, a component of diesel exhaust particles, Chem. Res. Toxicol., 15, 483-489, 2002.

Li, N., Sioutas, C., Cho, A. K., Schmitz, D., Misra, C., Sempf, J., Wang, M., Oberley, T., Froines, J., and Nel, A.: Ultrafine particulate pollutants induce oxidative stress and mitochondrial damage, Environ. Health Persp., 111, 455-460, 2003.

Li, Q. F., Wyatt, A., and Kamens, R. M.: Oxidant generation and toxicity enhancement of aged-diesel exhaust, Atmos. Environ., 43, 1037-1042, 2009.

Lin, P. and Yu, J. Z.: Generation of reactive oxygen species mediated by Humic-like Substances in atmospheric aerosols, Environ Sci. Technol., 45, 10362-10368, 2011. 
Mudway, I. S., Fuller, G., Green, D., Dunster, C., and Kelly, F. J.: Report: Quantifying the London specific component of $\mathrm{PM}_{10}$ oxidative activity, University of London, Defra, UK, 2011.

Nel, A.: Air pollution-related illness: effects of particles, Science, 308, 804-806, 2005.

Nel, A. E., Diaz-Sanchez, D., Ng, D., Hiura, T., and Saxon, A.: Enhancement of allergic inflammation by the interaction between diesel exhaust particles and the immune system, J. Allergy Clin. Immun., 102, 539-554, doi:10.1016/S0091-6749(98)70269-6, 1998.

Norris, G., YoungPong, S. N., Koenig, J. Q., Larson, T. V., Sheppard, L., and Stout, J. W.: An association between fine particles and asthma emergency department visits for children in Seattle, Environ. Health Persp., 107, 489-493, 1999.

Ntziachristos, L., Froines, J. R., Cho, A. K., and Sioutas, C.: Relationship between redox activity and chemical speciation of sizefractionated particulate matter, Particle and Fibre Toxicology, 4, 5, doi:10.1186/1743-8977-4-5, 2007.

Peter, M. and Steffen, L.: Oxidative damage to DNA and Lipids as biomarkers of exposure to air pollution, Environ. Health Persp., 118, 1126-1136, 2010.

Pope, C. A., Burnett, R. T., Thurston, G. D., Thun, M. J., Calle, E. E., Krewski, D., and Godleski, J. J.: Cardiovascular mortality and long-term exposure to particulate air pollution: epidemiological evidence of general pathophysiological pathways of disease, Circulation, 109, 71-77, 2004.

Sameenoi, Y., Koehler, K., Shapiro, J., Boonsong, K., Sun, Y., Collett, J., Volckens, J., and Henry, C. S.: Microfluidic electrochemical sensor for on-line monitoring of aerosol oxidative activity, J. Am. Chem. Soc., 134, 10562-10568, 2012a.

Sameenoi, Y., Panymeesamer, P., Supalakorn, N., Koehler, K., Chailapakul, O., Henry, C. S., and Volckens, J.: Microfluidic paper-based analytical device for aerosol oxidative activity, Environ. Sci. Technol., 47, 932-940, 2012b.

Samet, J. M., Dominici, F., Curriero, F. C., Coursac, I., and Zeger, S. L.: Fine particulate air pollution and mortality in 20 U.S. cities, 1987-1994, New Engl. J. Med., 343, 1742-1749, 2000.

Schaumann, F., Borm, P. J. A., Herbrich, A., Knoch, J., Pitz, M., Schins, R. P. F., Luettig, B., Hohlfeld, J. M., Heinrich, J., and Krug, N.: Metal-rich ambientparticles (particulate matter2.5) cause airway inflammation in healthy subjects, Am. J. Resp. Crit. Care, 170, 898-903, 2004.

Steenhof, M., Gosens, I., Strak, M., Godri, K. J., Hoek, G., Cassee, F. R., Mudway, I. S., Kelly, F. J., Harrison, R. M., Lebret, E., Brunekreef, B., Janssen, N. A. H., and Pieters, R. H. H.: In vitro toxicity of particulate matter (PM) collected at different sites in the Netherlands is associated with PM composition, size fraction and oxidative potential-the RAPTES project, Particle and Fibre Toxicology, 8, 26, doi:10.1186/1743-8977-8-26, 2011.

Sun, Q. H., Hong, X. R., and Wold, L. E.: Cardiovascular effects of ambient particulate air pollution exposure, Circulation, 121, 2755-2765, 2010.

Tong, H., Rappold, A. G., Diaz-Sanchez, D., Steck, S. E., Berntsen, J., Cascio, W. E., Devlin, R. B., and Samet, J. M.: Omega-3 fatty acid supplementation appears to attenuate particulate air pollution-induced cardiac effects and lipid changes in healthy middle-aged adults, Environ. Health Persp., 120, 952-957, 2012.
Turpin, B. J. and Lim, H.-J.: Species contributions to $\mathrm{PM}_{2.5}$ mass concentrations: revisiting common assumptions for estimating organic mass, Aerosol Sci. Tech., 35, 602-610, 2001.

Venkatachari, P., Hopke, P. K., Grover, B. D., and Eatough, D. J.: Measurement of particle-bound reactive oxygen species in rubidoux aerosols, J. Atmos. Chem., 50, 49-58, 2005.

Verma, V., Ning, Z., Cho, A. K., Schauer, J. J., Shafer, M. M., and Sioutas, C.: Redox activity of urban quasi-ultrafine particles from primary and secondary sources, Atmos. Environ., 43, 63606368, 2009a.

Verma, V., Polidori, A., Schauer, J. J., Shafer, M. M., Cassee, F. R., and Sioutas, C.: Physicochemical and toxicological profiles of particulate matter in Los Angeles during the October 2007 southern California wildfires, Environ. Sci. Technol., 43, 954960, 2009b.

Verma, V., Rico-Martinez, R., Kotra, N., King, L. E., Liu, J., Snell, T. W., and Weber, R. J.: Contribution of water-soluble and insoluble components and their hydrophobic/hydrophilic subfractions to the reactive oxygen species-generating ootential of fine ambient aerosols, Environ. Sci. Technol., 46, 11384-11392, 2012.

Verma, V., Fang, T., Guo, H., King, L., Bates, J. T., Peltier, R. E., Edgerton, E., Russell, A. G., and Weber, R. J.: Reactive oxygen species associated with water-soluble $\mathrm{PM}_{2.5}$ in the southeastern United States: spatiotemporal trends and source apportionment, Atmos. Chem. Phys., 14, 12915-12930, doi:10.5194/acp14-12915-2014, 2014.

Wilson, J. G., Kingham, S., Pearce, J., and Sturman, A. P.: A review of intraurban variations in particulate air pollution: Implications for epidemiological research, Atmos. Environ., 39, 6444-6462, 2005.

Xia, T., Kovochich, M., Brant, J., Hotze, M., Sempf, J., Oberley, T., Sioutas, C., Yeh, J. I., Wiesner, M. R., and Nel, A. E.: Comparison of the abilities of ambient and manufactured nanoparticles to induce cellular toxicity according to an oxidative stress paradigm, Nano Lett., 6, 1794-1807, 2006.

Zanobetti, A., Coull, B. A., Gryparis, A., Kloog, I., Sparrow, D., Vokonas, P. S., Wright, R. O., Gold, D. R., and Schwartz, J.: Associations between arrhythmia episodes and temporally and spatially resolved black carbon and particulate matter in elderly patients, Occup. Environ. Med., 71, 201-207, 2014.

Zhang, X., Hecobian, A., Zheng, M., Frank, N. H., and Weber, R. J.: Biomass burning impact on $\mathrm{PM}_{2.5}$ over the southeastern US during 2007: integrating chemically speciated FRM filter measurements, MODIS fire counts and PMF analysis, Atmos. Chem. Phys., 10, 6839-6853, doi:10.5194/acp-10-6839-2010, 2010.

Zomer, B., Collé, L., Jedyńska, A., Pasterkamp, G., Kooter, I., and Bloemen, H.: Chemiluminescent reductive acridinium triggering (CRAT) - mechanism and applications, Anal. Bioanal. Chem., 401, 2945-2954, 2011. 